\title{
THE LOGNORMAL-LIKE DISTRIBUTIONS IN THE LINEAR GROWTH EQUATIONS
}

\author{
NING-NING PANG* and WEN-JER TZENG ${ }^{\dagger}$ \\ *Department of Physics, National Taiwan University, Taipei, \\ Taiwan, Republic of China \\ ${ }^{\dagger}$ Department of Physics, Tamkang University, Tamsui, Taipei, \\ Taiwan, Republic of China
}

Received 8 August 2002

\begin{abstract}
The distributions of the global interfacial widths, correlation functions, and the local interfacial widths of the growth process described by the one-dimensional EdwardWilkinson equation are shown to be denumerable convolutions of exponential distributions. The same conclusions can also be extended to the distributions of the global interfacial widths for another linear growth equation, describing some super-rough growth processes, in both one- and two-dimensional cases. Most of these distributions display the lognormal-like behavior. We propose that the mechanism provided by the accumulation of exponential random variables may contribute to a lot of the lognormal-like behavior observed in the social and natural sciences.
\end{abstract}

Interfaces play an important role in a number of physical, chemical, and biological processes. The fluctuations of the quantities introduced to characterize these interfaces, such as the surface widths, correlation functions, and so on, display perceptible universal features. Accordingly, models mimicking interface motion have been analyzed and distinguished in terms of universality classes. ${ }^{1}$ The distributions of the global interfacial widths have been introduced to provide a detailed characterization of surface growth processes ${ }^{2,3}$ and they have been used to establish universality classes of rather diverse phenomena. ${ }^{4}$ An interesting feature of the distribution of the global interfacial width for the one-dimensional (1- $d$ ) EdwardsWilkinson $(\mathrm{EW})$ equation ${ }^{5}$ is that its short-time limit is practically identical to the lognormal distribution. ${ }^{3}$ Lognormal-like distributions tend to emerge more often in social, biological, atmospheric, and geological sciences than in physics ${ }^{6}$ and they are usually understood in terms of the law of proportionate effect or on the basis of the assumption that an event occurs only if a large number of independent subevents take place (like the theory of breakage). ${ }^{7}$

In this report, we will study the mechanism for the emergence of the lognormallike behavior. First of all, we observe that the distribution of the global interfacial width in the 1- $d$ EW equation is a denumerable convolution of exponential distributions (CED, usually called the Polya frequency function on $\mathbf{R}_{+}$of order $\infty$ in 
mathematics). ${ }^{8}$ In particular, it is a special case of the generalized gamma convolution $(\mathrm{GCG})^{9}$ and, of course, is infinitely divisible. Recall that the lognormal distribution is also infinitely divisible. ${ }^{10}$ We are motivated to introduce a quantitative measure of the resemblance between a given distribution and its lognormal fit, based on the ratios of the moments of the distributions. In the 1- $d$ case, we also consider some related quantities, the correlation function and the local interfacial width, ${ }^{11}$ which turn out to be denumerable CED, too. Moreover, their distributions at later times are closer to their lognormal fits based on the comparison of the ratios of the moments. We thus make a conjecture on the conditions for a denumerable CED to display the lognormal-like behavior. Finally, we check our conjecture on the distributions of the global interfacial widths of another linear growth equation, describing some super-rough growth processes, ${ }^{11}$ in both $1-d$ and 2 - $d$ cases.

The simplest equation describing the surface evolution governed by the surface tension and the noise is the EW equation: ${ }^{5}$

$$
\frac{\partial h(x, t)}{\partial t}=\nu \frac{\partial^{2}}{\partial x^{2}} h(x, t)+\eta(x, t),
$$

where $h(x, t)$ denotes the interface height at position $x$ and time $t, \nu$ is a constant related to the dynamical surface tension, and $\eta(x, t)$ represents the Gaussian white noise with zero mean and the correlation $\overline{\eta(x, t) \eta\left(x^{\prime}, t^{\prime}\right)}=2 \Gamma \delta\left(x-x^{\prime}\right) \delta\left(t-t^{\prime}\right)$. Here $0 \leq x<L$ and the periodic boundary condition is assumed. Throughout the paper, the overbar denotes the statistical average. By performing the Fourier transform $\eta(x, t)=\sum_{n=-\infty}^{\infty} \eta_{n}(t) e^{i k_{n} x}$ with $k_{n} \equiv \frac{2 \pi n}{L}, \eta_{-n}=\eta_{n}^{*}$, and denoting $\operatorname{Re} \eta_{n}\left(=\operatorname{Re} \eta_{-n}\right)$ by $\zeta_{n}$ and $\operatorname{Im} \eta_{n}\left(=-\operatorname{Im} \eta_{-n}\right)$ by $\xi_{n}$, it is straightforward to derive that $\overline{\zeta_{n} \zeta_{m}}=\overline{\xi_{n} \xi_{m}}=\frac{\Gamma}{L} \delta_{n, m}$ and $\overline{\zeta_{n} \xi_{m}}=0$, which hold for any integers $n$ and $m$ except $n=m=0\left(\overline{\zeta_{0} \zeta_{0}}=2 \Gamma / L\right.$ and $\left.\xi_{0}=0\right)$. Then, perform the Fourier transform $h(x, t)=\sum_{n=-\infty}^{\infty}\left(\alpha_{n}+i \beta_{n}\right)(t) e^{i k_{n} x}$, with real random variables $\alpha_{-n}=\alpha_{n}$ and $\beta_{-n}=-\beta_{n} \cdot{ }^{12}$ The EW equation in $k$-space is then obtained as

$$
\frac{d}{d t} \alpha_{n}(t)+\nu k_{n}^{2} \alpha_{n}(t)=\zeta_{n}(t) \quad \text { and } \quad \frac{d}{d t} \beta_{n}(t)+\nu k_{n}^{2} \beta_{n}(t)=\xi_{n}(t) .
$$

The solutions of Eq. (2) are $\alpha_{n}(t)=\int_{0}^{t} \zeta_{n}(t-\tau) e^{-\nu k_{n}^{2} \tau} d \tau+\alpha_{n}(0) e^{-\nu k_{n}^{2} t}$ and $\beta_{n}(t)=$ $\int_{0}^{t} \xi_{n}(t-\tau) e^{-\nu k_{n}^{2} \tau} d \tau+\beta_{n}(0) e^{-\nu k_{n}^{2} t}$. Since all $\eta(x, t)$ 's are Gaussian distributed, their linear combinations, $\zeta_{n}, \xi_{n}, \alpha_{n}$, and $\beta_{n}$, are thus also Gaussian distributed. All the distributions of $\zeta_{n}$ 's, $\xi_{n}$ 's, $\alpha_{n}$ 's, and $\beta_{n}$ 's can then be easily determined by knowing their first two moments. After some calculation, the probability densities of $\alpha_{n}$ and $\beta_{n}$ are then obtained as

$$
f_{\alpha_{n}}(x)=\frac{1}{\sqrt{2 \pi} \sigma_{n}} e^{-\frac{\left(x-\left\langle\alpha_{n}\right\rangle\right)^{2}}{2 \sigma_{n}^{2}}} \text { and } \quad f_{\beta_{n}}(x)=\frac{1}{\sqrt{2 \pi} \sigma_{n}} e^{-\frac{\left(x-\left\langle\beta_{n}\right\rangle\right)^{2}}{2 \sigma_{n}^{2}}},
$$

with $\left\langle\alpha_{n}(t)\right\rangle=\alpha_{n}(0) e^{-\nu k_{n}^{2} t},\left\langle\beta_{n}(t)\right\rangle=\beta_{n}(0) e^{-\nu k_{n}^{2} t}$, and $\sigma_{n}(t)=\sqrt{\frac{\Gamma\left(1-e^{-2 \nu k_{n}^{2} t}\right)}{L \nu k_{n}^{2}}}$. We will concentrate on the case with the flat initial condition, i.e. $\alpha_{n}(0)=\beta_{n}(0)=0$ for all $n \neq 0$. 
Next, the global interfacial width $w(L, t)$ is defined by $w^{2}(L, t) \equiv\langle(h(x, t)-$ $\left.\left.\langle h(x, t)\rangle_{L}\right)^{2}\right\rangle_{L}$ with $\langle\cdots\rangle_{L}$ denoting the lateral spatial average over the whole system of lateral size $L$. It can be shown that $w^{2}(L, t)=\sum_{n=-\infty}^{\infty}\left|\alpha_{n}+i \beta_{n}\right|^{2}-\mid \alpha_{0}+$ $\left.i \beta_{0}\right|^{2}=2 \sum_{n=1}^{\infty}\left(\alpha_{n}^{2}+\beta_{n}^{2}\right)$. Thus, the distribution of $w^{2}(L, t)$ is the convolution of the distributions of $\alpha_{n}^{2}$ and $\beta_{n}^{2}$ for all $n>0$. By applying Eq. (3), the probability densities of $\alpha_{n}^{2}$ and $\beta_{n}^{2}(n \neq 0)$ are obtained as

$$
f_{\alpha_{n}^{2}}(x)=f_{\beta_{n}^{2}}(x)=\frac{1}{\sqrt{2 \pi x} \sigma_{n}} e^{-\frac{x}{2 \sigma_{n}^{2}} x} \geq 0 .
$$

A crucial observation is that the probability density of the sum of $\alpha_{n}^{2}$ and $\beta_{n}^{2}$, given by

$$
f_{\alpha_{n}^{2}+\beta_{n}^{2}}(x)=\frac{1}{2 \sigma_{n}^{2}} e^{-x / 2 \sigma_{n}^{2}} x \geq 0,
$$

is an exponential distribution with the parameter $2 \sigma_{n}^{2}$. We have thus reached the conclusion that $w^{2}(L, t)$ has a Polya density on $\mathbf{R}_{+}$of order $\infty .^{8}$ Note that the expectation of $w^{2}(L, t)$ is $\overline{w^{2}(L, t)}=4 \sum_{n=1}^{\infty} \sigma_{n}^{2}(t)$ with the long time limit $\overline{w_{\infty}^{2}}=$ $\frac{\Gamma L}{6 \nu}$.

Subsequently, let us turn to the Laplace transform of the scaled distribution $\Phi(x) \equiv f_{w^{2} / \overline{w_{\infty}^{2}}}(x)=\overline{w_{\infty}^{2}} f_{w^{2}}\left(\overline{w_{\infty}^{2}} x\right)$, given by

$$
\mathcal{L}(s) \equiv \int_{0}^{\infty} e^{-s x} \Phi(x) d x=\prod_{n=1}^{\infty} \frac{1}{1+s a_{n}}
$$

with $a_{n}=\frac{6}{(n \pi)^{2}}\left(1-e^{-n^{2} \tau}\right)$ and $\tau=2 \nu\left(\frac{2 \pi}{L}\right)^{2} t$. Here, $\left\{a_{n} \mid 0<n<\infty\right\}$ is the set of parameters for the exponential distributions to compose the distribution $\Phi(x)$. In this formulation, we see that $\Phi(x)$ is a GCG since it matches the standard representation of GCG: ${ }^{9}$

$$
\mathcal{L}(s)=\exp \left[-a s+\int_{0}^{\infty} \ln \left(\frac{y}{y+s}\right) U(y) d y\right], \quad \text { for } a \geq 0 \text { and } s \in \mathbf{C} \backslash(-\infty, 0)
$$

with $a=0$ and $U(y)=\sum_{n=1}^{\infty} \delta\left(y-a_{n}^{-1}\right)$. The plots of $\Phi(x)$ at several different times and the corresponding lognormal fits are shown in Fig. 1. The parameters of the lognormal fits are chosen to have the same first and second moments as the distribution to be fitted (different from Ref. 3). We find that the fitting is much better at small $\tau$. Although the lognormal distribution is infinitely divisible, ${ }^{10}$ its form in the standard representation of GCG has not been found. We conjecture that the excellent fitting at short times is related to the clustering of atomic masses in the $U$-measure at short times since the values of $a_{n}$ 's coincide in the first order expansion with $n^{2} \tau$ as the small parameter. For further investigation, we will introduce some quantitative measures for the fitting through the comparison of the ratios of the moments. Since all $a_{n}$ 's are independent, the moments of $\Phi(x)$, defined 
as $M_{m}(\Phi) \equiv \int_{0}^{\infty} x^{m} \Phi(x) d x$, are given by

$$
\begin{aligned}
M_{1}(\Phi) & =A_{1}, \quad M_{2}=A_{2}+A_{1}^{2}, \quad M_{3}=2 A_{3}+3 A_{2} A_{1}+A_{1}^{3}, \\
M_{4} & =A_{4}+8 A_{3} A_{1}+3 A_{2}^{2}+6 A_{2} A_{1}^{2}+A_{1}^{4}, \ldots
\end{aligned}
$$

with $A_{m} \equiv \sum_{n=1}^{\infty} a_{n}^{m}$. In contrast, for a longnormal distribution with the probability density given by

$$
f_{\mathrm{LN}}(x)=\frac{1}{\sqrt{2 \pi} \sigma x} \exp \left[-\frac{(\ln x-\mu)^{2}}{2 \sigma^{2}}\right], \quad x \geq 0,
$$

the moments are given by

$$
M_{m}\left(f_{\mathrm{LN}}\right)=\exp \left(m \mu+\frac{1}{2} m^{2} \sigma^{2}\right) .
$$

The lognormal fit of $\Phi(x)$ is obtained by matching the first two moments, i.e.

$$
\mu=\frac{1}{2} \ln \frac{A_{1}^{4}}{A_{2}+A_{1}^{2}} \quad \text { and } \quad \sigma^{2}=\ln \frac{A_{2}+A_{1}^{2}}{A_{1}^{2}} .
$$

From Eq. (10), the next two moments of the lognormal fit turn out to be

$$
M_{3}\left(f_{\mathrm{LN}}\right)=\frac{\left(A_{2}+A_{1}^{2}\right)^{3}}{A_{1}^{3}} \quad \text { and } \quad M_{4}\left(f_{\mathrm{LN}}\right)=\frac{\left(A_{2}+A_{1}^{2}\right)^{6}}{A_{1}^{8}} .
$$

We then introduce the two ratios

$$
r_{3} \equiv \frac{M_{3}(\Phi)}{M_{3}\left(f_{\mathrm{LN}}\right)} \quad \text { and } \quad r_{4} \equiv \frac{M_{4}(\Phi)}{M_{4}\left(f_{\mathrm{LN}}\right)}
$$

as the quantitative measures for the fitting. The fitting is better as $r_{3}$ and $r_{4}$ are closer to one. We note that the lognormal density function is not uniquely determined by its moments, ${ }^{13}$ but it does not affect our results as seen directly from the fitting in Fig. 1.

As a comparison, we also calculate the distributions of the correlation function and the local interfacial width for the 1- $d$ EW equation. The correlation function $G(r, t)$ is defined as $G(r, t) \equiv\left\langle(h(x, t)-h(x+r, t))^{2}\right\rangle_{L}$ and the local width $\tilde{w}(l, t)$

Table 1. The ratios of the moments for the distributions of $w^{2}(L, t), G(r, t)$ and $\tilde{w}^{2}(l, t)$ in the $1-d$ EW equation at different times. Here, we choose $r / L=l / L=1 / 20$.

\begin{tabular}{ccccccc}
\hline & \multicolumn{2}{c}{$w^{2}(L, t)$} & \multicolumn{2}{c}{$G(r, t)$} & \multicolumn{2}{c}{$\tilde{w}^{2}(l, t)$} \\
$\tau$ & $r_{3}$ & $r_{4}$ & $r_{3}$ & $r_{4}$ & $r_{3}$ & $r_{4}$ \\
\hline 0.01 & 0.999687 & 0.998686 & 0.999272 & 0.997004 & 0.992601 & 0.971489 \\
0.04 & 0.998831 & 0.994885 & 0.998518 & 0.994024 & 0.988275 & 0.955062 \\
0.16 & 0.995862 & 0.980797 & 0.998154 & 0.992614 & 0.982887 & 0.933947 \\
0.64 & 0.983435 & 0.924611 & 0.997897 & 0.991626 & 0.979757 & 0.921025 \\
2.56 & 0.968705 & 0.868713 & 0.997816 & 0.991303 & 0.978585 & 0.917068 \\
10.24 & 0.968342 & 0.867429 & 0.997816 & 0.991303 & 0.978559 & 0.916983 \\
$\infty$ & 0.968343 & 0.867429 & 0.997816 & 0.991303 & 0.978559 & 0.916983 \\
\hline
\end{tabular}




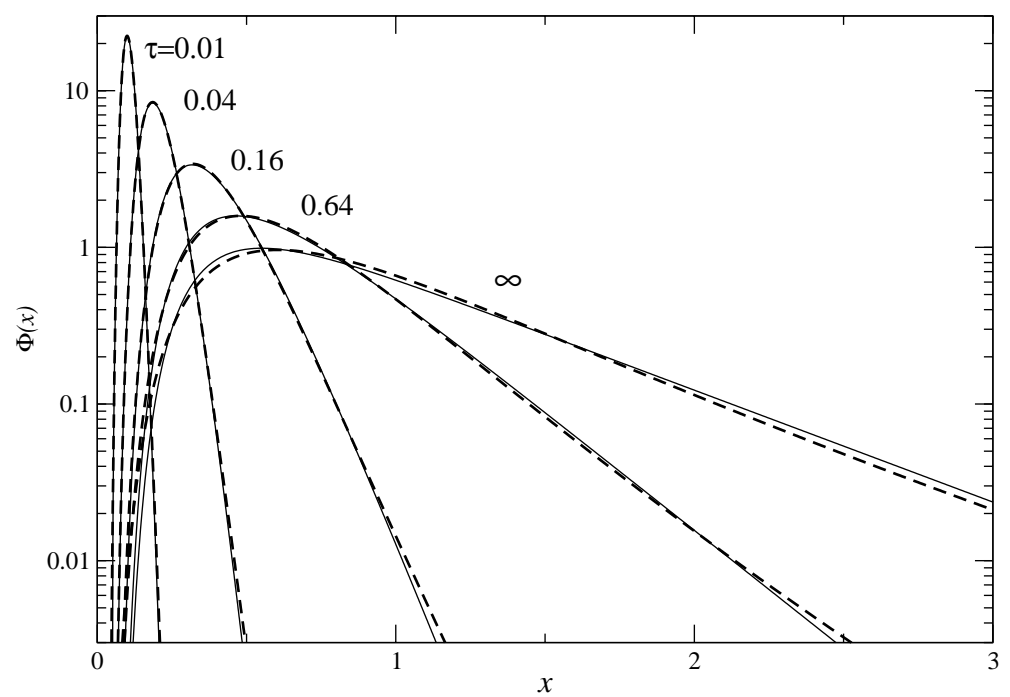

Fig. 1. The scaled distribution functions of the global interfacial width at different times for the 1- $d$ EW equation and their lognormal fits (dashed lines).

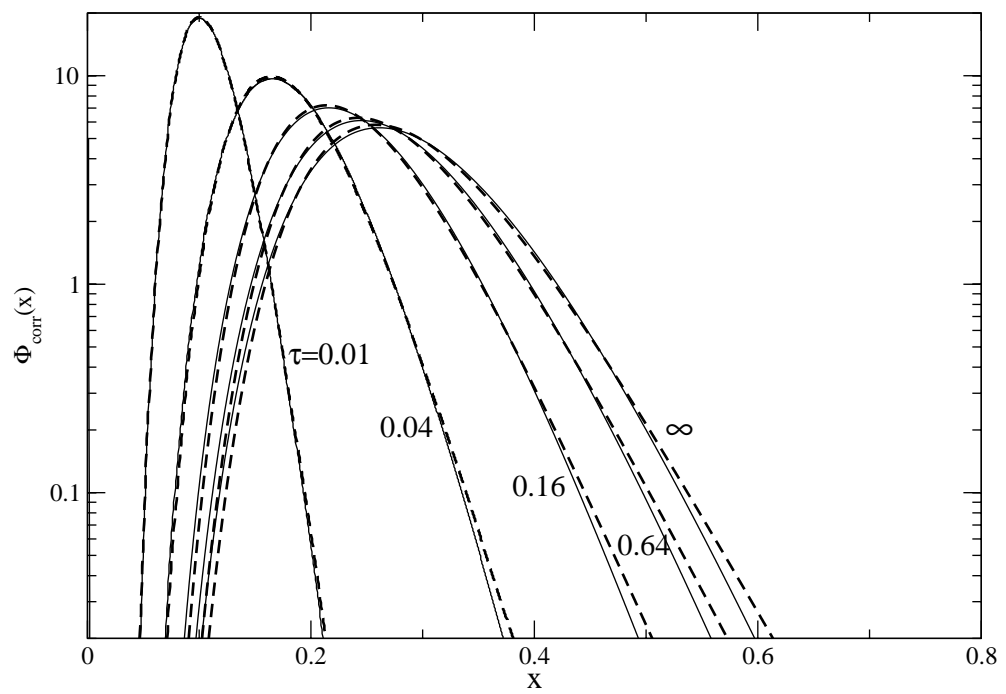

Fig. 2. The scaled distribution functions of the correlation function at different times for the 1-d EW equation and their lognormal fits (dashed lines).

is defined as $\tilde{w}^{2}(l, t) \equiv\left\langle\left\langle\left(h(x, t)-\langle h(x, t)\rangle_{l}\right)^{2}\right\rangle_{l}\right\rangle_{L}$ with $\langle\cdots\rangle_{l}$ denoting the lateral spatial average within a local window of lateral size $l$. After some calculation, ${ }^{11}$ we find that

$$
G(r, t)=4 \sum_{n=0}^{\infty}\left(\alpha_{n}^{2}+\beta_{n}^{2}\right)\left(1-\cos k_{n} r\right)
$$




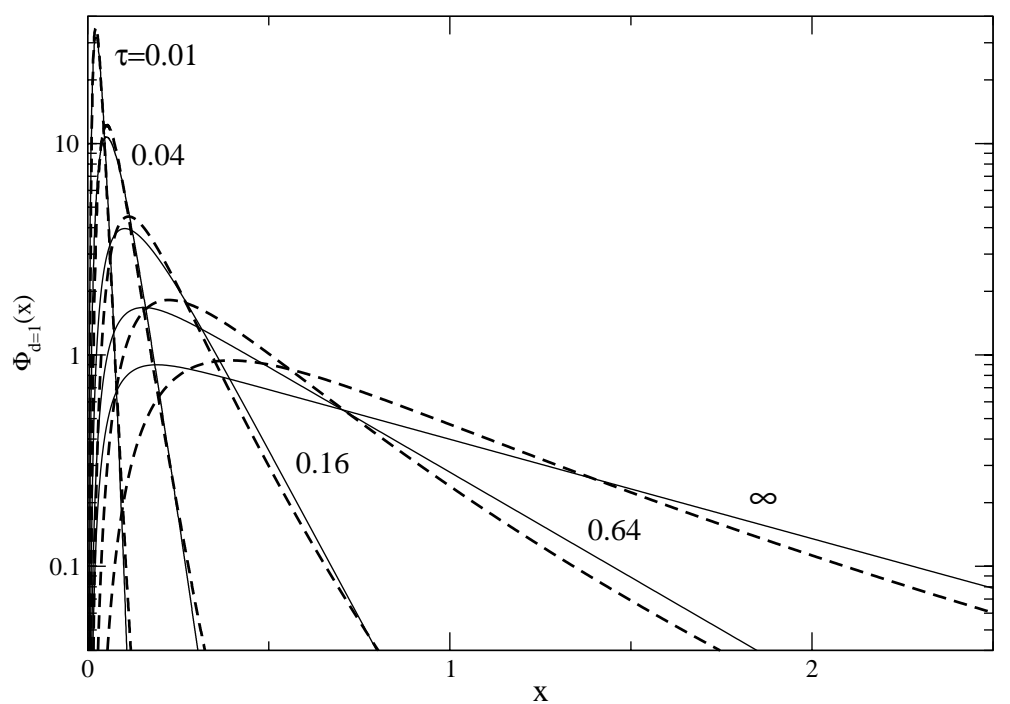

Fig. 3. The scaled distribution functions of the global interfacial width at different times for Eq. (16) and their lognormal fits (dashed lines).

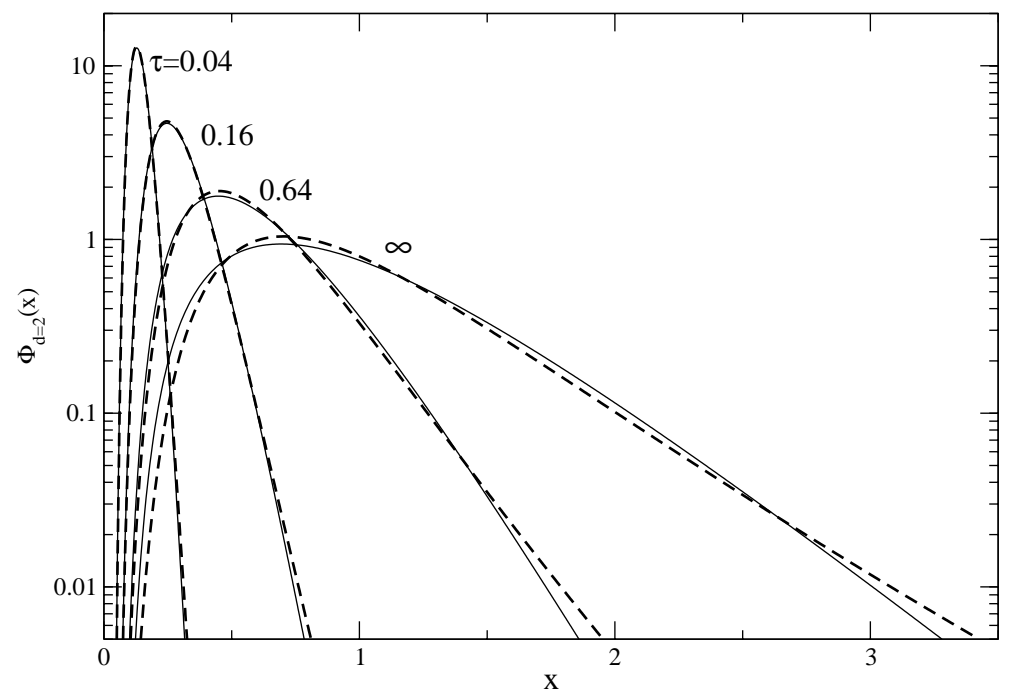

Fig. 4. The scaled distribution functions of the global interfacial width at different times for the 1- $d$ version of Eq. (16) and their lognormal fits (dashed lines).

and

$$
\tilde{w}^{2}(l, t)=4 \sum_{n=0}^{\infty}\left(\alpha_{n}^{2}+\beta_{n}^{2}\right)\left[\frac{1}{2}+\frac{1}{k_{n}^{2} l^{2}}\left(\cos k_{n} l-1\right)\right] .
$$

Thus, both distributions are still denumerable CED. Their distributions can be 
derived by exactly the same way as that for $w^{2}(L)$ except that the parameter $a_{n}$ is replaced by $\bar{a}_{n} \equiv\left(1-\cos \frac{2 \pi n r}{L}\right) a_{n}$ and by $\tilde{a}_{n}=\left[\frac{1}{2}+\frac{L^{2}}{4 \pi^{2} l^{2}}\left(\cos \frac{2 \pi n l}{L}-1\right)\right] a_{n}$, respectively. The scaled distributions of $G(r, t)$ at several different times are shown in Fig. 2. We see that the fitting is quite good at all times. To make a quantitative comparison, we collect the values of $r_{3}$ and $r_{4}$ for all $w^{2}(L, t), G(r, t)$, and $\tilde{w}^{2}(l, t)$ in Table 1. As expected, the two additional factors in $\bar{a}_{n}$ and $\tilde{a}_{n}$ favor the clustering of the values in the sets $\left\{\bar{a}_{n} \mid 0<n<\infty\right\}$ and $\left\{\tilde{a}_{n} \mid 0<n<\infty\right\}$ at later times, so the lognormal fits look better for $G(r, t)$ and $\tilde{w}^{2}(l, t)$ based on the values of $r_{3}$ and $r_{4}$.

Table 2. The ratios of the moments for the distributions of the global interfacial widths for the 1- $d$ and $2-d$ cases of Eq. (16) at different times.

\begin{tabular}{ccccc}
\hline & \multicolumn{2}{c}{$w^{2}(L, t)$ for $1-d$ Eq. $(16)$} & \multicolumn{2}{c}{$w^{2}(L, t)$ for $2-d$ Eq. $(16)$} \\
$\tau$ & $r_{3}$ & $r_{4}$ & $r_{3}$ & $r_{4}$ \\
\hline 0.01 & 0.975262 & 0.908476 & 0.999092 & 0.996309 \\
0.04 & 0.954055 & 0.837050 & 0.996738 & 0.986746 \\
0.16 & 0.918766 & 0.728530 & 0.989668 & 0.958176 \\
0.64 & 0.847570 & 0.549632 & 0.963229 & 0.863223 \\
2.56 & 0.807758 & 0.470860 & 0.943604 & 0.800967 \\
10.24 & 0.803917 & 0.463862 & 0.941286 & 0.793486 \\
$\infty$ & 0.803916 & 0.463860 & 0.941285 & 0.793482 \\
\hline
\end{tabular}

Finally, we would like to check the width distributions for the 2- $d$ case. However, the critical dimension for the EW equation is 2, so we turn to another linear growth equation describing some super-rough growth processes: ${ }^{11}$

$$
\frac{\partial h(x, y, t)}{\partial t}=-\nu\left(\frac{\partial^{4}}{\partial x^{4}}+\frac{\partial^{4}}{\partial y^{4}}\right) h(x, y, t)+\eta(x, y, t) \text {. }
$$

We solve both the 1- $d$ and $2-d$ versions of this equation. In the $1-d$ case, the scaled distribution of the global width is still described by Eq. (6) except that the parameters are given by $\hat{a}_{n} \equiv \frac{90}{(n \pi)^{4}}\left(1-e^{-n^{4} \tau}\right)$ and $\tau=2 \nu\left(\frac{2 \pi}{L}\right)^{4} t$. In the $2-d$ case, we set the lateral sizes $L_{x}=L_{y}=L$ for convenience and the scaled distribution of the global width is also described by Eq. (6) but the set of parameters $\left\{a_{n} \mid 0<n<\infty\right\}$ should now be replaced by $\left\{a_{n, m} \mid 0 \leq n<\infty, 0 \leq m<\infty\right.$ excluding $\left.n=m=0\right\}$ with $a_{n, m} \equiv \frac{33.28657}{\left(n^{4}+m^{4}\right) \pi^{4}}\left[1-e^{-\left(n^{4}+m^{4}\right) \tau}\right]$ and $\tau=2 \nu\left(\frac{2 \pi}{L}\right)^{4} t$. The corresponding results as those for the EW equation are shown in Figs. 3 and 4, and Table 2. As expected, since the distribution of $\hat{a}_{n}$ 's for the 1- $d$ case is sparse as compared to that for the EW equation, the fitting by the lognormal distribution is not as good as that for the EW equation. In the 2- $d$ case, there are more degrees of freedom in choosing $(n, m)$ and the distribution of $a_{n, m}$ 's becomes denser. The lognormal fitting is thus better than the 1- $d$ case.

In conclusion, we find that the distributions of the global interfacial width and other related quantities are, in fact, denumerable CED. Most of them fit quite well with their lognormal fits. Thus, we propose that the accumulation of independent 
exponential random variables, with their respective parameters appropriately distributed, may be responsible for lots of the lognormal-like behavior observed in the social and natural sciences. In the future work, we will proceed to explore the distributions of the parameters for the constitutive exponential random variables to compose a lognormal-like distribution.

This work is supported in part by grants from the National Science Council of Republic of China under the contract Nos. NSC-91-2112-M002-039 and NSC-912112-M032-005.

\section{References}

1. J. Krug, Adv. Phys. 46, 139 (1997).

2. G. Foltin, K. Oerding, Z. Rácz, R. L. Workman and R. K. P. Zia, Phys. Rev. E50, R639 (1994).

3. T. Antal and Z. Rácz, Phys. Rev. E54, 2256 (1996).

4. E. Marinari, A. Pagnani, G. Parisi and Z. Rácz, Phys. Rev. E65, 026136 (2002) and references therein.

5. S. F. Edwards and D. R. Wilkinson, Proc. R. Soc. (London) A381, 17 (1982).

6. See, for example, Lognormal Distributions: Theory and Applications, eds. E. L. Crow and K. Shimizu (Marcel Dekker, New York, 1988).

7. K. Shimizu and E. L. Crow, in Ref. 6.

8. S. Karlin, Total Positivity, Vol. I (Stanford Univ. Press, Stanford, 1968).

9. L. Bondesson, Generalized Gamma Convolutions and Related Classes of Distributions and Densities (Springer Verlag, New York, 1992).

10. O. Thorin, Scand. Actuarial J. 121 (1977).

11. N.-N. Pang and W.-J. Tzeng, Int. J. Mod. Phys. B26, 3429 (2001).

12. Some of the subsequent procedures can be found in Ref. 3. They are repeated here (with some modifications) because these derivations also work for other quantities which we introduce in this report.

13. C. C. Heyde, J. R. Statist. Soc. 25, 392 (1963); R. Leipnik, Theor. Probability Appl. 26, 850 (1981). 